\title{
Electroluminescence Properties of InGaN/AlGaN/GaN Light Emitting Diodes with Quantum Wells
}

\author{
A.E.Yunovich, V.E.Kudryashov, A..N.Turkin. \\ M.V.Lomonosov Moscow State University,Department of Physics, \\ A.N.Kovalev, F.I.Manyakhin. \\ Moscow Institute of Steel and Alloys, \\ Moscow, Russia. \\ E-mail: yunovich@scon175.phys.msu.su
}

\begin{abstract}
Electroluminescence spectra of light-emitting diodes based on InGaN/AlGaN/GaN heterostructures with single and multiple quantum wells (QWs) are analyzed by models of radiative recombination in 2D-structures with band tails. Equations of the model fit spectra quite good in a wide range of currents. Parameters of the fit are discussed and compared for single and multiple QWs. Tunnel effects play a sufficient role in blue LEDs with single QWs at low currents; they can be neglected in LEDs with multiple QWs. A new spectral band was detected at the high energy side of the spectra of green LEDs with multiple QWs; it is attributed with large scale inhomogenities of In distribution in InGaN QWs.*)
\end{abstract}

Keywords: light-emitting diodes, heterostructures, quantum wells, nitrides, luminescence.

*) This work proceeds previous results of our group presented on EGW-3 [1].

\section{Introduction}

The problems of recombination mechanisms in $\mathrm{InGaN} / \mathrm{AlGaN} / \mathrm{GaN}$ heterostructures are not fully understood in spite of great progress in the development of GaN-based light-emitting diodes (LEDs). We have studied luminescence spectra of two groups of LED's based on structures with single InGaN quantum wells (SQWs, sent to Moscow University by Dr. S.Nakamura, Nichia Chemical Co.) and multiple quantum wells (MQWs, sent to Moscow University by Dr. M.Koike, Toyoda Gosei Co.) in a wide range of currents [2-6]. A model of radiative recombination in 2Dstructures with band tails caused by potential fluctuations was successfully applied to describe 
the spectra [2-4]; tunnel radiative recombination was detected at low currents [5]. In this work spectra of blue and green LEDs with SQW and MQW active layers are compared and the model of recombination is analyzed. Changes of the parameters with current $\mathrm{J}$ and voltage $\mathrm{V}$ are discussed.
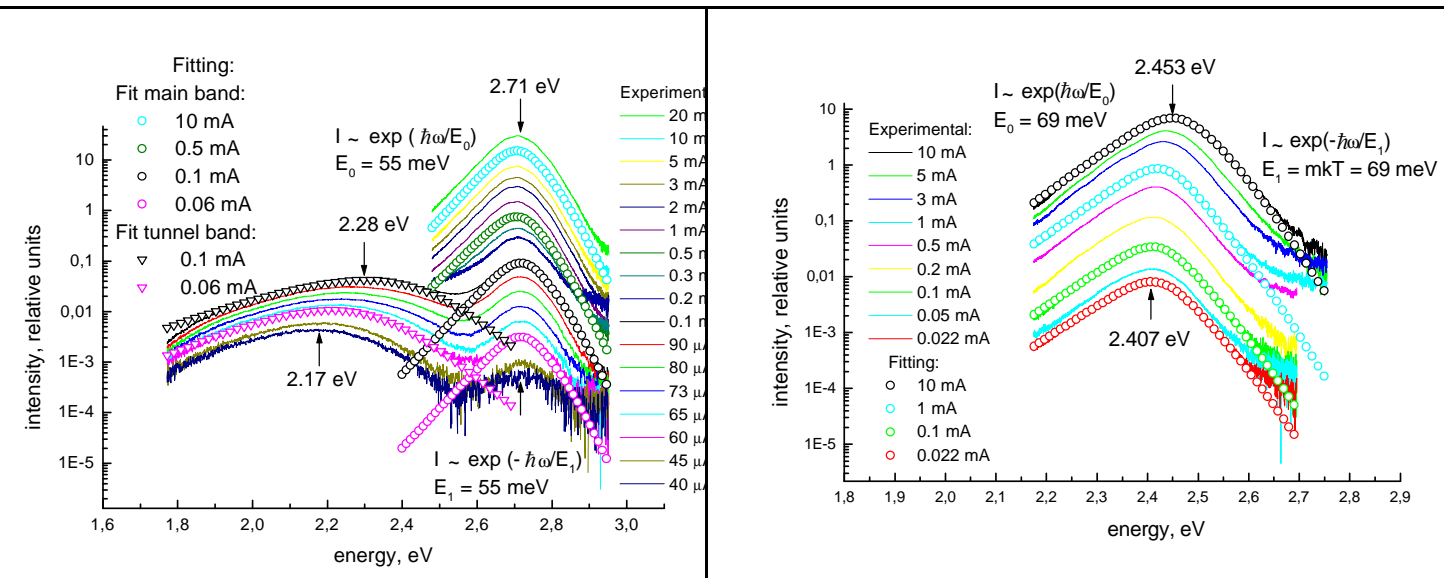

Figure 1a. Electroluminescence spectra of blue LED with SQW.

Figure 2a. Electroluminescence spectra of green LED with SQW.
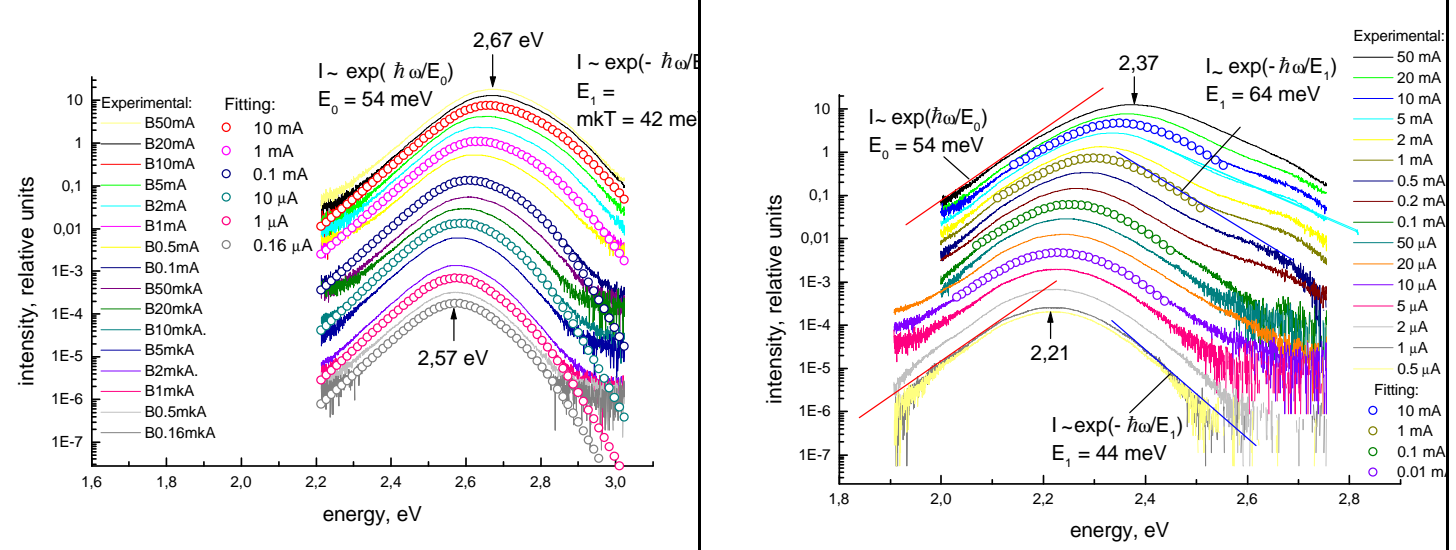

Figure 1b. Electroluminescence spectra of blue LED with MQW.

Figure 2b. Electroluminescence spectra of green LED with MQW.

\section{Experimental results.}

Both groups of LEDs were based on heterostructures grown on sapphire substrates by a MOCVD $[2,6]$. A buffer layer was of GaN in Nichia samples [2] and of AlN in Toyoda samples [6]. The base layer was $\mathrm{n}-\mathrm{GaN}: \mathrm{Si}(4-5 \mathrm{~mm})$.

The active layers of the structures were different: an $\mathrm{SQW} \operatorname{In}_{\mathrm{x}} \mathrm{Ga}_{1-\mathrm{x}} \mathrm{N}(>3.5 \mathrm{~nm})$ in Nichia LEDs and MQW $\operatorname{In}_{\mathrm{x}} \mathrm{Ga}_{1-\mathrm{x}} \mathrm{N} / \mathrm{GaN}$ (5 periods, a width of the period $<8 \mathrm{~nm}$ ). Indium content $\left(\mathrm{x}\right.$ in $\operatorname{In}_{\mathrm{x}} \mathrm{Ga}_{1-}$ ${ }_{x} \mathrm{~N}$ ) varied in both cases (near $\mathrm{x}=0.2$ for green and near $\mathrm{x}=0.4$ for blue LEDs). Upper layers $\mathrm{p}$ - 
AlGaN:Mg and cap layers $\mathrm{p}-\mathrm{GaN}: \mathrm{Mg}$ have hole concentrations in the range $\mathrm{p}=5.10^{17}-2.10^{18} \mathrm{~cm}^{-}$ ${ }^{3}$, higher for blue SQWs.

The spectra of blue and green LEDs with SQWs and MQWs are shown in a wide range of J (see Figs. 1, 2). The spectra of LEDs with SQWs could be measured down to $15 \mu \mathrm{A}$, that ones with MQWs to $0.15 \mu \mathrm{A}$. The cause of this difference is a tunnel component of nonradiative current most pronounced in blue LEDs with SQWs. Values of $\hbar \omega_{\max }(\approx 2.65 \mathrm{eV})$ for blue SQWs LEDs do not depend on $\mathrm{J}$.

There is another spectral band on the low energy side of the main blue line at low currents (see Fig. 1a). The maximum of this band is moving with the current $J$ and voltage on the active layer $\mathrm{U}: \hbar \omega_{\max }=\mathrm{eU}+\Delta$ (in the range $=2.1-2.3 \mathrm{eV}$ ). This band was described as a tunnel radiative recombination [4,5]. There is no tunnel band in blue LEDs with MQWs; in this case the blue line is moving with $\mathrm{V}$.

Values of $\hbar \omega_{\max }$ for green LEDs, both SQWs and MQWs, are moving with $\mathrm{J}$ and $\mathrm{V}$, but in a wider range for MQWs: $2.2 \div 2.45 \mathrm{eV}$. A new band was detected at the high energy side $(2.7 \div 2.8$ $\mathrm{eV}$ ) of the green line of MQWs (see Fig. 2b).

The main spectral lines have exponential tails: $\mathrm{I}\left(\hbar \omega_{\max }\right) \sim \exp \left(\hbar \omega_{\max } / \mathrm{E}_{0}\right)$ on the low energy side and $\mathrm{I}\left(\hbar \omega_{\max }\right) \sim \exp \left(-\hbar \omega_{\max } / \mathrm{E}_{1}\right)$ on the high energy side of the peaks. Values of $\mathrm{E}_{0}=50 \div 60 \mathrm{meV}$ did not depend on the current and temperature $\mathrm{T}$ in a wide range of $\mathrm{J}$. Values of $\mathrm{E}_{1}$ were proportional to $\mathrm{T}$ : $\mathrm{E}_{1}=\mathrm{mkT} ; 1<\mathrm{m}<2 ; \mathrm{m}$ is almost equal to unity for SQWs and is in the range $\mathrm{m}=1.5-1.7$ for MQWs. $\mathrm{E}_{1}$ was growing at higher direct currents, $\mathrm{J}>5 \mathrm{~mA}$, due to heating of the LEDs.



Figure 3. Distributions of charged centers in p-regions of p-n-heterojunctions; points - values of $\mathrm{N}_{\mathrm{A}}{ }^{-}$at $\mathrm{V}=0$; begining of abscissa is at the n-interface. Blue $(1,3)$ and green $(2,4-6)$ LEDs with SQW $(1,2)$ and MQW (3, 4-6). 


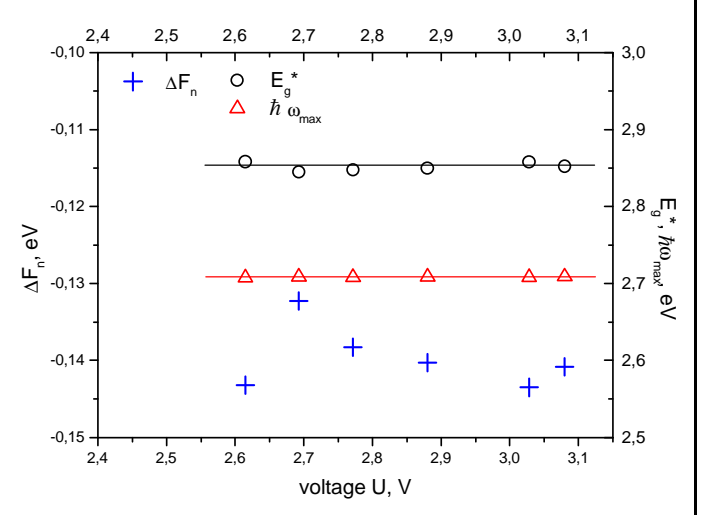

Figure 4a. Dependence of fitting parameters $\left(\Delta \mathrm{F}_{\mathrm{n}}, \mathrm{E}_{\mathrm{g}}{ }^{*}, \hbar \omega_{\max }\right)$ on the voltage for blue LED with SQW.

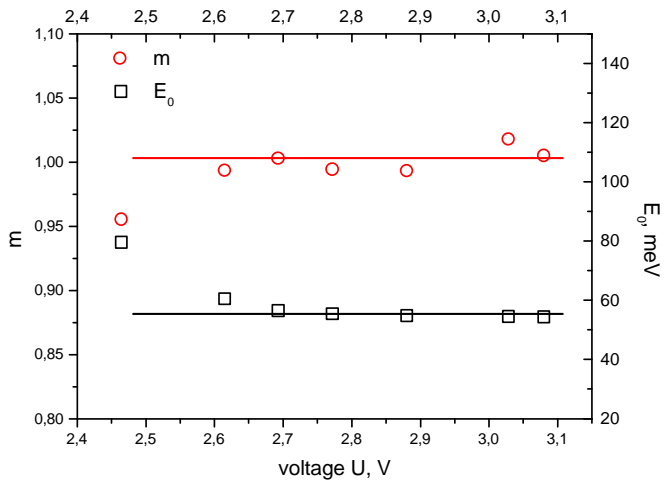

Figure 5a. Dependence of fitting parameters $\left(\mathrm{m}, \mathrm{E}_{0}\right)$ on the voltage for blue LED with SQW.

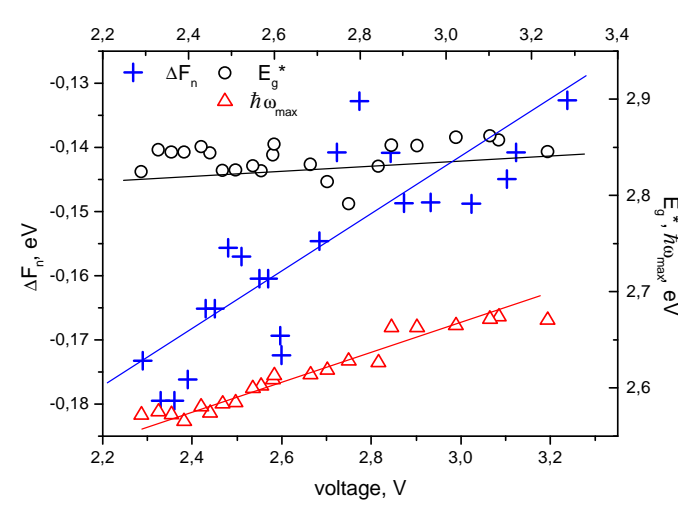

Figure 4b. Dependence of fitting parameters $\left(\Delta \mathrm{F}_{\mathrm{n}}, \mathrm{E}_{\mathrm{g}}{ }^{*}, \hbar \omega_{\max }\right)$ on the voltage for blue LED with MQW.

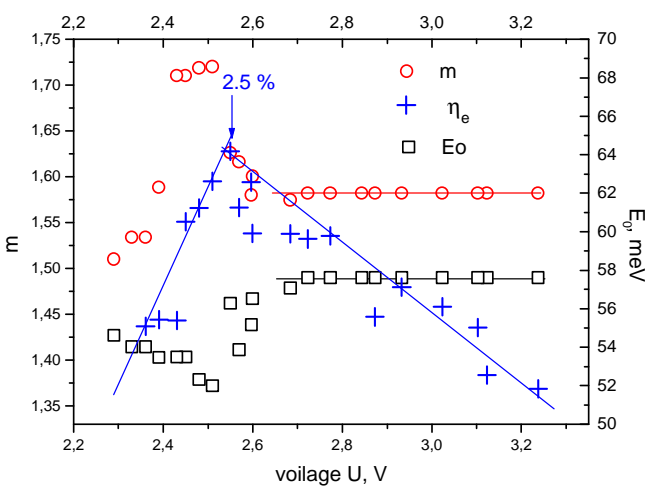

Figure 5b. Dependence of fitting parameters $\left(\mathrm{m}, \mathrm{E}_{0}\right)$ and quantum efficiency $\eta_{\mathrm{e}}$ on the voltage for blue LED with MQW.

Current-voltage characteristics $\mathrm{J}(\mathrm{V})$ of blue and green LEDs had an exponential part at low currents, $\mathrm{J}<10^{-7} \mathrm{~A}$ at $300 \mathrm{~K}$, a steep exponential growth in the range $\mathrm{V}=2.3-2.7 \mathrm{~V}$, a linear part at higher currents, $\mathrm{J}>20 \mathrm{~mA}$. Low currents could be understood as a tunnel component; tunnel currents in these LEDs play some role at J 3-4 orders of magnitude lower than that for SQWbased LEDs [2-5,7]; J(V) curves of SQW-based LEDs differed from MQW-based LEDs because of a wider active layer [1].

A good approximation of the J(V) curves for MQW LEDs was done when not only a series resistance $R_{s}$ at the linear part at higher $J$ was taken into account, but also the quadratic part: $J$ $\left(\mathrm{V}-\mathrm{V}_{1}\right)^{2}$. The fit of the curve $\mathrm{J}(\mathrm{V})$ at $\mathrm{J}>0.1 \mathrm{~mA}$ by the equation: 


$$
\mathrm{V}=\varphi_{\mathrm{k}}+\mathrm{E}_{\mathrm{J}} \cdot\left[\ln \left(\mathrm{J} / \mathrm{J}_{0}\right)+\left(\mathrm{J} / \mathrm{J}_{1}\right)^{0.5}\right]+\mathrm{J} \cdot \mathrm{R}_{\mathrm{s}}
$$

A part $\sim\left(\mathrm{J}_{\mathrm{J}}\right)^{0.5}$ is sufficient between an exponential (injection) and a linear parts, in the range $\mathrm{J}=2 \div 30 \mathrm{~mA}$, at usual working currents [1].

Distributions of charged centers in p-regions of InGaN/AlGaN/GaN p-n- heterostructures with MQWs and SQWs are shown, see Fig. 3 (see a method of measurements in [8]). The LEDs with MQWs have wider space charge width than ones with SQWs [2-5,7]; in both cases the width for green LEDs is wider than for blue ones. This fact corresponds to a low probability of tunnel effects in the LEDs with MQWs.

It seems that high $\mathrm{Mg}$-doping of $\mathrm{p}$ - $\mathrm{AlGaN}$ and $\mathrm{GaN}$ - layers is more difficult for higher In concentration in InGaN active layers.

\section{Discussion}

We describe the main lines in spectra with a model previously applied for fitting the spectra of LEDs with SQW [1-3]. The model implies that an effective radiative recombination takes place when carriers of both singes are injected into the active layer at voltages on the layer $U<V, U$ close to $\varphi_{\mathrm{k}}$. Optical transitions $\hbar \omega$ are going between states $\mathrm{E}_{(\mathrm{c})}$ and $\mathrm{E}_{(\mathrm{v})}$ in tails of $2 \mathrm{D}$-structure, caused by potential fluctuations. A model 2D- joint density of states is

$$
\mathrm{N}^{2 \mathrm{D}}\left(\hbar \omega-\mathrm{E}_{\mathrm{g}}^{*}\right)=\left[1+\exp \left(-\left(\hbar \omega-\mathrm{E}_{\mathrm{g}}^{*}\right) / \mathrm{E}_{0}\right)\right]^{-1}
$$

an effective energy gap $\mathrm{E}_{\mathrm{g}}^{*}=\mathrm{E}_{\mathrm{g}}(\mathrm{x}, \mathrm{T})+\Delta \mathrm{E}_{1 \mathrm{c}}+\Delta \mathrm{E}_{1 \mathrm{v}}+\Delta \mathrm{E}_{\mathrm{p}}-\mathrm{E}_{\mathrm{exc}}-\Delta \mathrm{E}_{\mathrm{D}, \mathrm{A}}$, where $\mathrm{E}_{\mathrm{g}}(\mathrm{x}, \mathrm{T})$ - the energy bandgap in the active layer $-\mathrm{QW}, \Delta \mathrm{E}_{1 \mathrm{c}}, \Delta \mathrm{E}_{1 \mathrm{v}}-$ quantum size energy levels in conduction and valence bands, $\Delta \mathrm{E}_{\mathrm{p}^{-}}$lattice deformation shift, $\mathrm{E}_{\mathrm{exc}^{-}}$energy of $2 \mathrm{D}$ exciton, $\Delta \mathrm{E}_{\mathrm{D}, \mathrm{A}^{-}}$donor-aceptor potential shift of the effective bandgap. The values $\mathrm{E}_{\mathrm{g}}^{*}$ received from this model are 2.98-3.05 eV for blue and 2.67-2.76 eV for green LED [3]. Spectral intensity $\mathrm{I}(\hbar \omega)$ is proportional to Fermi-functions of electrons and holes with quasi-Fermi levels $F_{n}, F_{p}$ as parameters (details in [2,3]):

$$
\mathrm{I}(\hbar \omega) \sim \mathrm{N}^{2 \mathrm{D}}\left(\hbar \omega-\mathrm{E}_{\mathrm{g}}^{*}\right) \mathrm{f}_{\mathrm{c}}\left(\hbar \omega, \mathrm{m}, \mathrm{kT}, \mathrm{F}_{\mathrm{n}}\right)\left(1-\mathrm{f}_{\mathrm{v}}\left(\hbar \omega, 1-\mathrm{m}, \mathrm{kT}, \mathrm{F}_{\mathrm{p}}\right)\right)
$$

Examples of the fit are shown in Figs. 2, 3. A change of $\hbar \omega_{\max }$ in some range of $\mathrm{J}$ is caused mostly by changes of the parameter $\mathrm{F}_{\mathrm{n}}$; states in the tails are filled as $\mathrm{V}$ and $\mathrm{J}$ are growing (see Fig. 4). Parameters of the fit $\mathrm{E}_{\mathrm{g}}{ }^{*}, \mathrm{E}_{0}, \mathrm{E}_{1}=\mathrm{m} \cdot \mathrm{kT}$ can be seen in Figs. 4, 5 for blue MQWs and SQW. There is the evidence that a mechanism of recombination in the 2D-tail of states is not changed in some range. It is remarkable that when this mechanism begins the quantum efficiency $\eta_{\mathrm{e}}$ reaches a maximum (see Fig. $5 b$ ). When the states in the active layers are filled by current carriers at high $\mathrm{J}$, the quantum efficiency $\eta_{\mathrm{e}}$ gradually falls.

There is a change of mechanism of recombination at lower currents; this can be concluded from Fig. $5 \mathrm{~b}$ in spite of scattering of the points. Comparing spectra in the Figs. 1a and $1 \mathrm{~b}$ we assume that this change is somewhat connected with tunneling.

A change of mechanism of recombination at higher currents in green LEDs, pronounced as a new band in Fig. 2b, can be connected with nonuniform distribution of Indium in the active layers InGaN more probable at higher In content. 


\section{Conclusions}

Mechanism of radiative recombination in 2D- structures with band tails describe the main line in the spectra of both groups blue and green LEDs, with SQWs and MQWs, in a wide range of currents. The main difference in spectra of two groups is a role of tunnel effects at low currents in LEDs with SQWs. Moving of spectra with the voltage is more pronounced in green LEDs due to higher content of Indium and more pronounced density of states tails in the active InGaN layers.

\section{Acknowledgments}

Authors thank Dr. S.Nakamura (Nichia Chemical Co.) and Dr. M.Koike (Toyoda Goasei Co.) for sending LEDs to Moscow University; S.S.Mamakin and R.V.Grigoryev for help in measurements.

\section{References}

[1] Yunovich A.E., Kudryashov V.E., Turkin A.N., A.N.Kovalev, F.I.Manyachin The Emission Properties of Light Emitting Diodes utilising Multiple Quantum Wells InGaN/AlGaN/GaN. GaN MRS Intern. Journ.of Nitride Semic. Res., $3 \backslash 44$.

[2] Zolina K.G., Kudryashov V.E., Turkin A.N., Yunovich A.E., Nakamura S., Luminescense Spectra of Superbright Blue and Green InGaN/AlGaN/GaN Light-Emitting Diodes, MRS Int. J. Of Nitride Semicond. Res. 1 (1996) 11; J. of the European Ceramic Society 17 (1997) p.2033.

[3] Zolina K.G., Kudryashov V.E., Turkin A.N., Yunovich A.E., Luminescense spectra of blue and green InGaN/AlGaN/GaN light-emitting diodes, Semiconductors. 31 (9) (1997) p. 1055.

[4] A. Yunovich, A.Kovalev, F. Manyachin, V. Kudryashov, A.N.Turkin, K.G.Zolina. Mechanisms of electroluminescence in InGaN/AlGaN/GaN heterojunctions with quantum wells. Proc. of the Second Symposium on III-V Nitride Materials and Processes, Vol.98-02 Electrochem. Soc., Pennington, NJ, 1998, pp.83-102.

[5] Kudryashov V., Zolina K., Turkin A., Yunovich A., Kovalev A., Manyakhin F., Tunneling effects in light-emitting diodes based on InGaN/AlGaN/GaN heterostructures with quantum wells, Semiconductors. 31 (11) (1997) p. 1123.

[6] Koike M., Koide N., Asami S., Umezaki J., Nagai S., Yamasaki S., Shibata N., Amano H., Akasaki I., GaInN/GaN multiple quantum wells green LEDs, Proc. of SPIEIntern.Soc.Opt.Engin. 3002 (1997).

[7] A.E.Yunovich, A.N.Kovalev, V.E.Kudryashov, F.I.Manyachin, A.N.Turkin, K.G.Zolina, "Tunnel Effects in Luminescence Spectra of InGaN/AlGaN/GaN Light-Emitting Diodes", MRS Symposium Proc., 449, 1167 (1997).

[8] F.I.Manyakhin, A.N.Kovalev, V.E.Kudryashov, A.N.Turkin, A.E.Yunovich, Semiconductors, 33 (1), (1999, to be published). 